\title{
Epileptiform movements associated with re-exposure to propofol
}

\section{EDITOR:}

Abnormal epileptiform activity related to anaesthesia with propofol is well described. In most of the cases these movements are seen on first exposure to propofol. We report a case of a patient who had previously undergone anaesthesia with propofol without any problems. However, on re-exposure to propofol the patient experienced severe and prolonged seizure-like activity that lasted for more than 4 days, which is likely to be related to the use of propofol.

A 22-yr-old female was admitted as a day case for extraction of symptomatic wisdom teeth. At the preoperative visit she was noted to be fit and healthy with no significant history of cardiovascular, respiratory or neurological disease. She reported that she had undergone two previous uneventful operations. The first was $5 \mathrm{yr}$ ago for an appendicetomy. For this procedure she was anaesthetized with thiopental and tracheal intubation was facilitated with the use of succinylcholine. Further muscle relaxation was maintained with atracurium. Analgesia was provided with fentanyl and morphine. The second operation occurred $2 \mathrm{yr}$ ago and was for a minor gynaecological procedure. For this procedure she was anaesthetized with a combination of propofol and fentanyl. Both anaesthetics were uneventful and there were no problems noted during recovery after either operation. She was not receiving medication and had no known drug allergies.

Full monitoring was instituted before the induction of anaesthesia. Propofol $200 \mathrm{mg}$ was used to induce anaesthesia, fentanyl to provide analgesia and mivacurium to facilitate nasotracheal intubation. Anaesthesia was maintained with nitrous oxide $70 \%$ in oxygen and isoflurane. Other drugs administered were granisetron, dexamethasone and ampicillin. The surgeon performed dental nerve blocks using lidocaine $2 \%$ with epinephrine 1 in 200000 . The dental extractions were completed uneventfully and once

Correspondence to: Sarang Puranik, Specialist Registrar in Anaesthetics and Pain, St. Peter's Hospital, Guildford Lane, Chertsey, KT16 0PZ, UK. E-mail: saranganita@ntlworld.com

Accepted for publication January 2003 EJA 1151 the patient was breathing satisfactorily the nasotracheal tube was removed and the patient sent to recovery to fully awaken. However, soon after waking up the patient started to have uncontrollable epileptiform movements of all her limbs with some opisthotonus. These episodes lasted for up to 3 min each and occurred about every $5 \mathrm{~min}$; they were not associated with urinary incontinence or tongue biting. No cyanosis was noted but on two occasions her oxygen saturation fell to $83 \%$ with tachycardia (119 beats $\mathrm{min}^{-1}$ ) The patient was not drowsy between the episodes although she was unresponsive during the 'fit'. The 'fits' were initially treated with increments of diazepam i.v. (total dose $15 \mathrm{mg}$ ). This treatment was of limited success although over the ensuing $2-4 \mathrm{~h}$ the episodes became less frequent and of shorter duration. The patient was returned to the ward for further observations overnight. During the night the episodes continued despite the use of diazepam $5 \mathrm{mg}$. The following day she was noted to be less responsive between episodes and she was transferred to our intensive care unit. Both a computerized tomographic scan and magnetic resonance imaging of the patient's brain revealed no abnormality. Electroencephalography during several of the 'fits' showed no evidence of epileptiform activity and serum prolactin concentration was unchanged during and following the 'fits'. Serum sodium, potassium, magnesium and creatinine concentrations and full blood count were normal. The patient remained apyrexial throughout the postoperative period. She was given a loading dose of phenytoin although this was later discontinued following advice from a neurologist. The episodes continued for a further 2 days and eventually ceased 5 days after the exposure to propofol. The patient was followed up 4 weeks after discharge and had had no further symptoms.

This patient had 'fits' for a prolonged time after a short surgical procedure. She was not a known epileptic beforehand. The 'fits' were shown to be nonepileptic and no other cause of these episodes was identified despite extensive investigations. The most likely cause is related to her exposure to propofol. 
The case is interesting for two reasons: firstly, the patient had previously been exposed to propofol and had had no untoward reaction, and secondly, the 'fits' lasted for an exceptionally long time after the exposure to propofol. Convulsions during or shortly after propofol anaesthesia have been reported a number of times since its introduction in 1986. In general, these reports described typically of twitching followed by a tonic clonic phase for a period of a few seconds or minutes followed by spontaneous recovery. The spectrum of neuroexcitatory events included, opisthotonus, muscle rigidity, confusion and athetoid movements. The onset of symptoms is variable, from a few seconds after propofol to $6 \mathrm{~h}$, but usually in the postoperative period. The duration and frequency of the seizures varies from single attacks lasting $10 \mathrm{~s}$ to repeated episodes continuing in one patient up to 23 days $[1,2]$.

It is still controversial whether propofol is proor anticonvulsant. In a recent systematic review presented at the annual meeting with the Austrian International Congress in Vienna, the authors found that there was a lack of evidence for propofol being pro- or anticonvulsant [3]. There are reports of various cases where it has been used as an anticonvulsant in intractable status epilepticus [4]. Various theories have been put forward for its different actions. Propofol is known to act by activating GABA receptors and enhancing its transmission [5]. In high concentration propofol depresses both cortex and subcortex acting as an anticonvulsant and in low concentration it may act as proconvulsant by inhibiting the inhibitory subcortex, resulting in the 'release' of normal hyperexcitability in the cortex. In the spinal cord it seems to act as a glycine antagonist like strychnine, which might explain the opisthotonus and myoclonus effects.

Interesting studies about EEG changes in healthy patients receiving propofol have been reported. Brainstem auditory evoked responses are dose-related with increased latencies as anaesthesia deepens. These changes are more akin to the volatile agents than other i.v. anaesthetics, but are too small to interfere with deafness testing when propofol is used for sedation. Seifert and colleagues found dose-related activation of $\beta$-frequencies of the EEG occurs with the onset of sedation and accompanying restlessness and agitation [6]. These changes persist with deepening sedation even though the excitatory movements stop - but cease within $30 \mathrm{~min}$ of discontinuing the propofol infusion. Borgeat and colleagues recorded the EEG during induction and saw activation with predominantly $\beta$-activity, without burst suppression, but with a transient period of $d$-waves coinciding with the movements. In the absence of EEG activity they concluded that the spontaneous movements have a subcortical origin. They also noted that the anti-emetic and antipruritic actions of low doses of propofol lasted much longer than hypnotic effects, indicating different actions at cortical and subcortical levels.

In our patient the most likely cause points towards propofol. This case is similar to others previously reported in that the patient received co-medication with fentanyl (as discussed by Hewitt and colleagues [7]). Other precipitating factors include hypocarbia, hypoxia, hyponatremia, pyrexia, but normal clinical findings and laboratory tests ruled out these possibilities. Amongst other drugs used during the procedure, fentanyl has been associated with tonicclonic grand mal seizures in the absence of any EEG changes but they have been seen during administration of high doses. Isoflurane is not reported to be epileptogenic in human beings in the clinically applied concentrations. Lidocaine is capable of producing seizures but again in toxic dose ranges. Seizures have been reported following penicillin injection. There have been few reports of extrapyramidal side-effects and possible convulsions following administration of the 5-HT antagonist ondansetron, but granisetron has been found to have a good safety profile. The most common side-effects reported with granisetron are headache, diarrhoea and asthenia. Thus, it would seem unlikely that any of the other drugs used in this case are involved in seizures. Normal computerized tomographic scan and magnetic resonance scan ruled out any organic causes such as space occupying lesion or haemorrhage.

It is important to control seizures in any case to avoid serious damage due to hypoxia, loss of airway control, regurgitation and pulmonary aspiration. Early involvement of a neurologist is advised. Control of 'seizures' can be achieved with benzodiazepines and if necessary with phenytoin. Close supervision of the patient is thus very important. This case highlights a very rare but potentially harmful complication of a very commonly employed i.v. anaesthetic agent in current use. Above all it should be noted that prior uneventful administration of propofol does not always rule out the possibility of such a complication on re-exposure.

$$
\begin{array}{r}
\text { S. Puranik } \\
\text { St. Peter's Hospital } \\
\text { Chertsey, UK } \\
\text { N. Quiney } \\
\text { Royal Surrey County Hospital } \\
\text { Surrey, UK }
\end{array}
$$

\section{References}

1. Bevan J. Propofol related convulsions. Can J Anesth 1993; 40: 805-809.

2. Collier C, Kelly K. Propofol and convulsions - the evidence mounts. Anaesth Intens Care 1992; 19: 573-575. 
3. Walder B, Seeck M, Tramèr MR. Pro- and anticonvulsant effects of propofol: a systematic review. Eur J Anaesthesiol 2000; 17 (Suppl 19): A272, 84.

4. Mackenzie SJ, Kapadia P, Grant IS. Propofol infusion for control of status epilepticus. Anaesthesia 1990; 45: 1043-1045.

5. Sneyd J. Excitatory events associated with propofol anaesthesia: a review. J Roy Soc Med 1992: 85: 288-290.
6. Seifert HA, Blouin RT, Conard PF, Gross JB. Sedative doses of propofol increase beta activity of the processed electroencephalogram. Anesth Analg 1993; 76: 976-978.

7. Hewitt PB, Chu DLK, Polkey CE and Binnie CD. Effect of propofol on the electrocorticogram in epileptic patients undergoing cortical resection. Br J Anaesth 1999; 82: 199-202.

\section{Laparoscopic live donor nephrectomy: the anaesthesiologist's perspective}

\section{EDITOR:}

Although kidney transplantation is nowadays considered the best therapeutic option for end-stage renal failure, organ shortage still represents the primary limiting factor. The average waiting time for a cadaveric kidney transplant at our institution was $3 \mathrm{yr}$ in 1990 and is now $8-10$ yr. Thus, there is renewed interest in alternative ways of increasing the supply of donors including the use of expanded criteria donors, non-heart-beating donors and living donors [1]. Since the first report of successful laparoscopic live donor nephrectomy in 1995 [2], this procedure, initially restricted to a few pioneer surgeons, is currently practised in a growing number of transplant centres throughout the world.

We report a single-centre experience for laparoscopic donor nephrectomy for living related kidney transplantation. We reviewed the charts of all laparoscopic kidney donations and the related transplants performed between April 2000 and December 2001 and compared this population with that of a corresponding number of traditional surgery donors from our historical series. Left nephrectomy was used because of anatomical advantages about the length of the renal vessels. The procedures were performed by two surgeons with the aid of a robotic arm to hold the endoscope (AESOP $3000^{\circledR}$ robotic visualization system; Computer Motion, Santa Barbara, CA, USA).

The anaesthesia technique was the same for both donors and recipients. After induction with fentanyl $0.2 \mathrm{mg}$, sodium thiopental $3 \mathrm{mg} \mathrm{kg}^{-1}$ and succinylcholine $1 \mathrm{mg} \mathrm{kg}^{-1}$, anaesthesia was maintained with sevoflurane in a $50 \%$ air/oxygen low-flow respiratory mixture; atracurium in a continuous intravenous (i.v.) infusion $\left(0.01 \mathrm{mg} \mathrm{kg}^{-1} \mathrm{~min}^{-1}\right)$ was used to

Correspondence to: Gianni Biancofiore, UTI Trapianti, Ospedale Cisanello, Via Paradisa 2, I-56100 Pisa, Italy. E-mail: g.biancofiore@med.unipi.it; Tel: +39 050 996885/996815; Fax: +39050996984

Accepted for publication December 2002 EJA 1273 achieve neuromuscular blockade. Remifentanil was given i.v. $\left(0.15 \mu \mathrm{g} \mathrm{kg}^{-1} \mathrm{~min}^{-1}\right)$. Because peritoneal $\mathrm{CO}_{2}$ insufflation can decrease blood flow to the kidneys (resulting in transient intraoperative kidney dysfunction and higher incidence of delayed graft function) [3], our laparoscopic donors received extraintravascular volume loading (saline $0.9 \% 2 \mathrm{~L}$ ) from the night before surgery. Intraoperatively, a positive fluid balance was maintained by colloids/crystalloids at a dose, exceeding surgical losses, of $10 \mathrm{~mL} \mathrm{~kg}^{-1} \mathrm{~h}^{-1}$; during the procedure, donor urine output of at least $100 \mathrm{~mL} \mathrm{~h}^{-1}$ was targeted, and loop diuretics or mannitol were also used to achieve this. Standard intraoperative monitoring, including arterial pressure and central venous pressure, was instituted. After completion of surgery, all donors were transferred to the intensive care unit.

We analysed the data using SPSS ${ }^{\circledR}$ v.7.0 (SPSS Inc., Chicago, IL, USA). The significance of the betweengroup differences was assessed using $t$-, $U$ - or $\chi^{2}$-tests as appropriate; $P=0.05$ was considered as statistically significant. During the study period, 28 living donor laparoscopic nephrectomies were performed; they were compared with 27 donors whose kidneys were harvested by a traditional flank approach. No procedure required conversion to laparotomy, nor did any donor develop complications. No patients needed re-intervention, and all the surgical procedures were uneventful. Laparoscopic donors underwent a longer procedure with a lower estimated blood loss but intraoperatively received more i.v. fluids; they needed less postoperative analgesia with a shorter intensive care unit stay and overall hospitalization; they also showed a quicker return to solid oral intake and full return to work (Table 1).

Monitored cardiovascular variables were stable in both groups, the open donors showing lower central venous pressures at anaesthesia induction and at the end of surgery; they also had significantly lower $\mathrm{PCO}_{2}$ throughout the entire procedure. Those recipients who received a traditionally harvested graft were 
Table 1. Donors' intraoperative and early recovery data.

\begin{tabular}{lcccc}
\hline & $\begin{array}{l}\text { Laparoscopic } \\
(\boldsymbol{n}=28)\end{array}$ & $\begin{array}{l}\text { Open } \\
(\boldsymbol{n}=27)\end{array}$ & $\boldsymbol{P}$ \\
\hline Anaesthesia time (min) & $255.4 \pm 34.8$ & $213 \pm 27$ & $<0.01$ \\
Estimated blood loss (mL) & $104 \pm 44$ & $301 \pm 113$ & 0.0001 \\
Intraoperative intravenous fluids (mL) & $2999 \pm 414$ & $2201 \pm 260$ & $<0.0001$ \\
ICU length of stay (h) & $11.9 \pm 5.3$ & $25.5 \pm 8.5$ & $<0.0001$ \\
Hospitalization (days) & $1.5 \pm 0.2$ & $5.6 \pm 1.4$ & $<0.001$ \\
Analgesics (tramadol) (mg) & $128 \pm 46.8$ & $714 \pm 179.1$ & $<0.0001$ \\
Resume oral intake (days) & $0.5 \pm 0.1$ & $2.4 \pm 0.2$ & $<0.01$ \\
Return to work (weeks) & $2 \pm 0.9$ & $5.9 \pm 2.8$ & $<0.001$ \\
\hline
\end{tabular}

Data are mean $\pm \mathrm{SD}$.

more often females ( 6 versus $1 ; P<0.05$ ) but did not differ for age $(32.3 \pm 8.8$ versus $30.7 \pm 8.2 \mathrm{yr}$; $P=0.6)$. Twenty-two procedures were single kidney transplants, whereas the remaining six were combined cadaveric pancreas and living donor kidney transplants.

Diuresis resumed intraoperatively in all recipients irrespectively of the donation approach; early graft function did not differ among the two groups, although serum creatinine concentration declined earlier, but not significantly, in the recipients of kidneys procured by the open method: both the recipient groups reached their nadir serum creatinine by postoperative day 14 . This difference did not affect the length of hospitalization or the ultimate graft function $(P=0.4)$; no recipient and no graft was lost. There was no significant difference in the number of dialysis treatments during recipients hospitalization $(P=0.5)$.

Live donor kidney transplants have been associated with several major advantages over cadaveric transplants: avoidance of long waiting times for the recipient, superior human leukocyte antigen matching, shorter cold ischaemia duration and significant improvement of graft survival rate [3]. Donor nephrectomy is unique among major surgical procedures as it exposes an otherwise healthy subject to the risks of a major surgical procedure without any personal benefit. Laparoscopic living kidney donation, as a still expanding technique, has to meet two utmost conditions: the laparoscopic donor, when compared with the 'open' approach, should suffer no additional morbidity due to the technique itself and harvested kidneys must have, at least, the same survival and function quality of those obtained with the standard extraperitoneal flank approach. In our experience, laparoscopic renal donation offers several advantages over the traditional approach in the postoperative period: lower analgesic requirements, faster resumption of food intake, shorter hospitalization, quicker return to work and a better postoperative cosmetic appearance (particularly attractive for younger donors). A very important aspect of laparoscopic renal donation is possible alteration in the graft's function because of intra-abdominal hypertension from the pneumoperitoneum [4]. It has been suggested that the critical intra-abdominal pressure necessary to promote renal dysfunction is $15 \mathrm{mmHg}$ [5]; however, this also represents the standard abdominal insufflation pressure usually reached during laparoscopic procedures. In this setting, although fluid administration in excess to the physiological need was reported as 'protective' [6], relative oliguria was observed soon after transplantation of laparoscopic donor kidneys independently from an aggressive intraoperative fluid loading [7] probably because renal dysfunction due to intraabdominal hypertension is related to a number of different factors [5]; thus, volume loading alone may not be effective. To avoid renal damage, a multidisciplinary approach is common practice at our centre, which is based on three key points: to keep laparoscopic donors with a positive fluid balance before and during the procedure; to obtain an urinary output of at least $100 \mathrm{mLh}^{-1}$ from the start of the procedure; and to inflate the donor's abdomen with an pressure not exceeding $12 \mathrm{mmHg}$.

The current discrepancy between potential recipients and kidney availability has led transplant physicians to search for new ways to provide more transplants; today, the most interesting option is living donation. Laparoscopic nephrectomy offers donors better postoperative comfort, faster recovery and better cosmetic results when compared with the traditional flank incision technique with the same graft's survival without higher or dedicated anaesthetic cost.

$$
\begin{array}{r}
\text { G. Biancofiore, G. Amorese, D. Lugli, } \\
\text { L. Bindi, N. Fossati } \\
\text { Azienda Ospedaliera Pisana, Department No. } 1 \\
\text { Anaesthesia and Intensive Care } \\
\text { Ospedale di Cisanello } \\
\text { Pisa, Italy }
\end{array}
$$




\section{Ugo Boggi, A. Pietrabissa, F. Mosca \\ General and Transplantation Surgery University School of Medicine Ospedale di Cisanello Pisa, Italy}

\section{References}

1. Thiel G. Living kidney donor transplantation, new dimensions. Transpl Int 1998; 11: 50-56.

2. Ratner LE, Cisek LJ, Moore LG, Cigarroa FG, Kaufman HS, Kavoussi LR. Laparoscopic live donor nephrectomy. Transplantation 1995; 60: 1047-1049.

3. D'Alessandro MA, Sollinger HW, Knechtle SJ, et al. Living related and unrelated donors for kidney transplantation. A 28-year experience. Ann Surg 1995; 222: 353-364.
4. Kirsch AJ, Hensle TW, Chang DT, Kayton ML, Olsson CA, Sawczuk IS. Renal effects of $\mathrm{CO}_{2}$ insufflation: oliguria and acute renal dysfunction in a rat pneumoperitoneum model. Urology 1994; 43: 453-459.

5. Sugrue M, Buist MD, Hourihan F, Deane S, Bauman A, Hillman K. Prospective study of intra-abdominal hypertension and renal function after laparotomy. Br J Surg 1995; 82: 235-238.

6. London ET, Ho HS, Neuhaus AM, Wolfe BM, Rudich SM, Perez RV. Effect of intravascular volume expansion on renal function during prolonged $\mathrm{CO}_{2}$ pneumoperitoneum. Ann Surg 2000; 231: 195-201.

7. Flowers JL, Jacobs S, Cho E, et al. Comparison of open and laparoscopic live donor nephrectomy. Ann Surg 1997; 226: 483-489.

\section{Perioperative neutropenia}

\section{EDITOR:}

Neutropenia and/or pancytopenia developing during operation, or soon afterwards, may pose a diagnostic challenge to anaesthesiologists. Interventions, such as blood transfusion (transfusion associated graft vs. host disease) or drugs, such as anaesthetics (nitrous oxide) or antibiotics (cephalosporins) as well as patient factors, such as paroxysmal nocturnal haemoglobinuria or aplastic anaemia, can cause neutropenia or pancytopenia [1-7].

A 31-yr-old female was admitted to the Anaesthesiology Intensive Care Unit (ICU) after maxillary reconstruction with flap operation. Adenocarcinoma had been diagnosed in 1997. She was not receiving any medication before operation; her preoperative laboratory tests were normal, including total white blood cell count of 5600 cells $\mathrm{mm}^{-3}$, haemoglobin $10.7 \mathrm{gm} \mathrm{dL}^{-1}$, platelets $239000 \mathrm{dL}^{-1}$. The patient's trachea was intubated using a fibrescope after propofol $200 \mathrm{mg}$, succinylcholine $50 \mathrm{mg}$, cefazolin $1 \mathrm{~g}$ and fentanyl $50 \mu \mathrm{g}$ had been given intravenously (i.v.). General anaesthesia was maintained with sevoflurane $2-3 \%$ in $\mathrm{O}_{2}$ and $2 \mathrm{~L} \mathrm{~min}^{-1}$ and $\mathrm{N}_{2} \mathrm{O} 4 \mathrm{~L} \mathrm{~min}^{-1}$; vecuronium was used for further muscle paralysis. Cefazolin $1 \mathrm{~g}$ was repeated twice intraoperatively and was continued postoperatively $1 \mathrm{~g}$ three times per day.

The intraoperative course during $7 \mathrm{~h}$ of surgery was especially notable for blood loss (estimated to be approximately $1800 \mathrm{~mL}$ ) which necessitated the administration of $4 \mathrm{U}$ of non-irradiated packed red blood cells, fresh frozen plasma $2 \mathrm{U}$, crystalloids $5 \mathrm{~L}$, hydroxyethylstarch $500 \mathrm{~mL}$. Intraoperatively, multiple complete blood counts were ordered (Table 1).

Correspondence to: Seda Akinci, Guzaltan sok No: 4/3 Birlik apt, Maltepe, Ankara 06570, Turkey. E-mail: sedabanu@yahoo.com; Fax: +90 3123109600

Accepted for publication January 2003 EJA 1401
The patient arrived in the Anaesthesiology ICU awake, well oxygenated and with a stable cardiovascular system. During the first postoperative day, her white cell count and haemoglobin concentration progressively declined and reached a nadir on the third postoperative day. Serum lactate dehydrogenase (peaked at $1975 \mathrm{UL}^{-1}$ ) and bilirubin concentration (peak total bilirubin $24.5 \mathrm{mg} \mathrm{dL}^{-1}$, peak direct bilirubin $19.38 \mathrm{mg} \mathrm{dL}^{-1}$ ) progressively increased together with dark urine, strongly positive for bilirubin. A peripheral smear revealed hypochromic, micrositic anisocytosis with $40 \%$ polymorph nuclear leucocytes, $52 \%$ atypical lymphocytes and $0.8 \%$ monocytes. Fine needle bone marrow aspiration showed fibrosis and aplastic bone marrow. Four units of antibody and subgroup cross-matched irradiated packed red blood cells were given. Prothrombin time, International Normalized Ratio, activated prothrombin time, serum haptoglobulin concentrations, serum folic acid and vitamin $\mathrm{B}_{12}$ concentrations were normal. Serum fibrinogen 747 (normal 144-430 $\mathrm{mg} \mathrm{dL}^{-1}$ ) and D-dimer concentrations $6.4\left(0.0-0.5 \mathrm{ng} \mathrm{dL}^{-1}\right)$ were elevated, whereas thrombin time $14.2(15-22 \mathrm{~s})$ and antithrombin III activity $72(80-120 \%)$ were low. Direct and indirect Coombs tests were negative. Other laboratory findings included the following: alanine aminotransferase $18 \mathrm{UL}^{-1}$, aspartate aminotransferase $59 \mathrm{UL}^{-1}$ and alkaline phosphatase $140 \mathrm{UL}^{-1}$.

On the third postoperative day spiked fever developed with a maximum body temperature of $38.8^{\circ} \mathrm{C}$ and neutropenic fever was diagnosed. Results of blood, sputum, wound, stool and urine cultures were negative. Serums for viral titres (cytomegalovirus, Epstein-Barr virus and hepatitis B virus) were not indicative of acute or past infection. The haematology department was consulted and bone marrow biopsy showed fatty tissue with rare mature bone 
Table 1. Perioperative complete blood count changes.

\begin{tabular}{|c|c|c|c|}
\hline Day & $\begin{array}{l}\text { White cell } \\
\text { count } \\
\left(\text { cells } \mathrm{mm}^{-3}\right)\end{array}$ & $\begin{array}{l}\text { Haemoglobin } \\
\text { concentration } \\
\left(\mathrm{g} \mathrm{dL}^{-1}\right)\end{array}$ & $\begin{array}{l}\text { Platelet } \\
\text { concentration } \\
\left(\text { cells } \mathrm{dL}^{-1}\right)\end{array}$ \\
\hline Preoperative & 5600 & 10.7 & 239000 \\
\hline \multirow[t]{3}{*}{ Intraoperative (total cefazolin $3 \mathrm{~g}$ ) } & 1300 & 8.8 & 152000 \\
\hline & 800 & 5.2 & 101000 \\
\hline & 2300 & 9.0 & 142000 \\
\hline Anaesthesiology ICU (cefazolin $1 \mathrm{~g} \times 3$ ) & 2500 & 12.7 & 140000 \\
\hline Postoperative day 1 & 1700 & 8.6 & 80000 \\
\hline Postoperative day 2 & 1200 & 8.4 & 78000 \\
\hline Postoperative day 3 & 500 & 6.3 & 90000 \\
\hline $\begin{array}{l}\text { Postoperative day } 3 \text { (cefazolin discontinued, } \\
\text { granulocyte colony-stimulating factor begun) }\end{array}$ & 500 & 5.1 & 82000 \\
\hline Postoperative day 4 & 700 & 8.0 & 86000 \\
\hline $\begin{array}{l}\text { Postoperative day } 5 \text { (granulocyte colony- } \\
\text { stimulating factor discontinued) }\end{array}$ & 11600 & 9.1 & 77000 \\
\hline Postoperative day 6 & 7500 & 8.8 & 79000 \\
\hline Postoperative day 7 & 9100 & 8.6 & 83000 \\
\hline
\end{tabular}

marrow elements. Granulocyte colony-stimulating factor $\left(30 \mathrm{mU} \mathrm{day}^{-1}\right)$ was administered i.v. together with amikacin $\left(1 \mathrm{~g} \mathrm{day}^{-1}\right)$ and cefepime $(2 \times 2 \mathrm{~g})$. Serum bilirubin and lactate dehydrogenase concentrations progressively decreased to normal while her white cell count and haemoglobin concentration increased. Antibiotics were discontinued and she was transferred to the plastic surgery ward on the eighth postoperative day and from the hospital on the eighteenth postoperative day.

In our patient, the decrease in haemoglobin concentration and white cell and platelet counts, and the peripheral smear findings and aplastic bone marrow all suggested the diagnosis of acute acquired aplastic anaemia. Acquired aplastic anaemia can be idiopathic or secondary to radiation, paroxysmal nocturnal haemoglobinuria, pregnancy, many classes of pharmaceutical agents, viruses or immune diseases [1]. Transfusion associated graft vs. host disease, paroxysmal nocturnal haemoglobinuria and drugs (nitrous oxide, antibiotic therapy) were probably the cause of the acquired aplastic anaemia.

Paroxysmal nocturnal haemoglobinuria is a rare acquired disorder of bone marrow stem cells resulting in the production red cells, granulocytes and platelets with both membrane and enzyme defects causing abnormal sensitivity to complement fixation [2]. These patients are particularly at risk for haemolysis and thrombosis as a result of surgery and anaesthesia $[2,3]$. We first entertained the diagnosis of paroxysmal nocturnal haemoglobinuria when our patient developed Coombs negative haemolysis and pancytopenia with a hypoplastic bone marrow but then had to exclude it because of the normal Ham's (acidified serum) and sucrose haemolysis tests.
Transfusion associated graft vs. host disease may occur in presumed immunocompetent patients (patients after cardiac surgery) receiving non-irradiated blood products [4]. Clinical features include pancytopenia, fever, pruritus, rash, abnormal results of liver function tests, dyspnoea and gastrointestinal symptoms such as nausea, vomiting, anorexia and diarrhoea [4]. Our patient complained of nausea, vomiting and abdominal discomfort that we thought were common postoperative problems. We suspected the diagnosis of acute graft vs. host disease after considering the chronology of events (blood transfusion, elevated aspartate aminotransferase, bilirubin concentration, pancytopenia with aplastic bone marrow) and so we gave only irradiated blood products after operation as irradiation of blood products is recommended for the prevention of this disease. But there was no rash or pruritus, and, fortunately, our patient improved after granulocyte colony-stimulating therapy and survived; therefore, we thought diagnosis of acute graft vs. host disease in our patient was unlikely.

The possibility of nitrous oxide induced bone marrow suppression cannot be entirely excluded [7]; however, it is extremely unlikely that this could have occurred only $1 \mathrm{~h}$ after nitrous oxide administration. In fit patients undergoing elective surgery, a period of at least 5-6h exposure to $50 \%$ nitrous oxide in 50\% oxygen is required before megaloblastic changes in bone marrow occur [7]. The white cell count did not return to normal after cessation of nitrous oxide, vitamin $B_{12}$ and folic acid concentrations were normal and the bone marrow did not show any megaloblastic changes [7].

Neutropenia seems to be related to the use of cefazolin sodium in our patient. Neutropenia secondary 
to antibiotic administration is thought to result primarily from an immunological reaction with latent period of 7-14 days after the first exposure or immediately after re-exposure $[5,6]$. The precipitous fall in white cell count within $2 \mathrm{~h}$ of cefazolin administration suggested prior exposure; and old medical records documented the use of cefazolin for prophylaxis in the previous operation. But, our patient did not have the rash, pruritus or eosinophilia that is common in antibiotic associated neutropenia. As a result, we would like to point out that anaesthesiologists should be aware that cefazolin can cause perioperative neutropenia. Not only haemoglobin and platelets but also haematological indices, such as white blood cell counts, should be monitored when cefazolin is given for surgical prophylaxis in patients undergoing major surgery.

$$
\begin{array}{r}
\text { S. B. Akinci, T. Aykut, A. Gozacan, } \\
\text { M. Kanbak, S. Ozgen, Ü. Aypar } \\
\text { Department of Anesthesiology and Reanimation } \\
\text { Hacettepe University, } \\
\text { Ankara, Turkey }
\end{array}
$$

\section{References}

1. Young NS. Acquired aplastic anemia. JAMA 1999; 282: 271-278.

2. Ogin GA. Cholecytectomy in a patient with paroxysmal nocturnal hemoglobinuria: anesthetic implications and management in the perioperative period. Anesthesiology 1990; 72: 761-764.

3. Kathirvel S, Prakash A, Lokesh BN, Sujatha P. The anesthetic management of a patient with paroxysmal nocturnal hemoglobinuria. Anesth Analg 2000; 91: 1029-1031.

4. Arsura EL, Bertelle A, Minkowitz S, Cunningham JN, Grob D. Transfusion-associated-graft-versus-host disease in a presumed immunocompetent patient. Arch Intern Med 1988, 148: 1941-1948.

5. Thompson JR, Garber R, Ayers H, Oki J. Cefazolinassociated neutropenia. Clin Pharmacy 1987; 6: 811-814.

6. Murphy MF, Chapman JF, Metcalfe P, Waters AH. Cephalosporin induced neutropenia. Br J Haematol 1986; 62: 394-397.

7. Gilman MA. Haematological changes caused by nitrous oxide. Cause for concern? Br J Anaesth 1987; 59: 143-144.

\section{Anaesthesia for a life-limited child with adrenoleucodystrophy}

\section{EDITOR:}

We present the anaesthetic management of a lifelimited 5-yr-old male with childhood-onset X-linked adrenoleucodystrophy who required general anaesthesia for insertion of a percutaneous endoscopic gastrostomy tube. Adrenoleucodystrophy is an X-linked recessive disorder with an incidence of about 1/100 000 and is characterized by the accumulation of saturated very long chain fatty acids leading to progressive dysfunction of the adrenal cortex and cerebral white matter. Patients usually present with progressive neurocognitive changes; however, around $9 \%$ of children can present acutely with adrenal crisis, seizures or encephalopathy [1].

Four months previously the patient had presented to the Accident and Emergency Department with status epilepticus and hypoglycaemia. Despite standard emergency treatment, admission to the Paediatric Intensive Care Unit was required for management of persistent seizures and recurrent hypoglycaemia. Following discharge to the general ward, subsequent investigation revealed a diagnosis of adrenoleucodystrophy, complicated by brain injury secondary

Correspondence to: Giles Dobson, Department of Anaesthesia, The Royal Group Hospitals, Grosvenor Road, Belfast, BT12 6BA, Northern Ireland. E-mail: gtdobson@hotmail.com; Tel: +35302890 240503; Fax: +35302890 325725

Accepted for publication February 2003 EJA 1398 to hypoxaemia and hypoglycaemia. The patient suffered episodic pulmonary aspiration due to progressive weakness of the bulbar muscles and a percutaneous endoscopic gastrostomy tube was considered necessary to facilitate long-term enteral feeding.

Preoperative assessment revealed a $25 \mathrm{~kg}$ male who was confined to a wheelchair and had limited interaction with his environment. He demonstrated abnormal involuntary hemiballismic movements (i.e. throwing of the limbs of one side of the body) and continuously drooled saliva due to his inability to swallow. He had excessive skin pigmentation due to adrenal dysfunction but physical examination was otherwise unremarkable. Preoperative medication included tetrabenzine, hydrocortisone, fludrocortisone, chloral hydrate, baclofen and dantrolene. The perioperative anaesthetic plan needed to encompass the risks of further seizure activity, adrenal insufficiency, pulmonary aspiration and the potential for altered drug pharmacokinetics and pharmacodynamics, particularly in relation to the use of sedative, opioid and muscle relaxant drugs.

Ranitidine was given as premedication. Standard monitoring was attached and after obtaining intravenous access, hydrocortisone $50 \mathrm{mg}$ was administered. Following aspiration of the nasogastric feeding tube, the patient was pre-oxygenated, cricoid pressure 
was applied and anaesthesia was induced with oxygen and incremental sevoflurane - to a maximum inspired concentration of $8 \%$. No muscle relaxant was administered and the patient continued to breathe spontaneously. The trachea was intubated with a $5.5 \mathrm{~mm}$ uncuffed endotracheal tube, without difficulty with direct laryngoscopy, and a gas leak was present at $20 \mathrm{cmH}_{2} \mathrm{O}$. The tube was secured and anaesthesia was maintained with sevoflurane, $\mathrm{O}_{2}\left(\mathrm{FiO}_{2}=0.5\right)$ and $\mathrm{N}_{2} \mathrm{O}$. Acetaminophen (paracetamol) $20 \mathrm{mg} \mathrm{kg}^{-1}$ was administered per rectum and further analgesia was achieved by infiltration of the surgical incision with local anaesthetic solution. After insertion of the percutaneous endoscopic gastrostomy tube, the patient's stomach was decompressed via the endoscope and once the oropharynx had been suctioned, he was turned into the lateral position and the trachea extubated. In the recovery unit, although the patient refused to tolerate $\mathrm{O}_{2}$ by mask, $\mathrm{S}_{\mathrm{P}} \mathrm{O}_{2}$ remained within normal limits during air breathing. After meeting discharge criteria, the patient was returned to the ward and monitored uneventfully using pulse oximetry overnight. Subsequently, the patient was discharged from hospital to await hospice care.

Although the anaesthetic management for patients with other leucodystrophies has been previously reported [2-4], this is the first report describing the anaesthetic management for a patient with childhoodonset X-linked adrenoleucodystrophy. The differences between the leucodystrophies are subtle and previous reports have highlighted the common concerns for the anaesthetist including mental retardation, seizure activity, hypotonia, abnormal liver function, pulmonary aspiration, adrenal insufficiency, perioperative temperature homeostasis and the potential for postoperative respiratory complications.

Patients with leucodystrophy have an increased incidence of gastro-oesophageal reflux, which may be secondary to altered gastric muscle tone [4]. Previous reports have recommended that a rapid sequence induction with cricoid pressure should be used. It is recognized that succinylcholine can cause massive hyperkalaemia in patients with neuromuscular disease [5]; however, there are no reports describing its use in this patient group. Therefore, it has been suggested that the rapid sequence induction could be modified by selecting a non-depolarizing muscle relaxant as a safer alternative. Our patient had been prescribed dantrolene $5 \mathrm{mg}$ every $8 \mathrm{~h}$ orally in a further effort to control muscle spasms. Dantrolene can cause prolonged neuromuscular blockade when administered in combination with a non-depolarizing muscle relaxant [6] and dantrolene-induced muscle weakness can lead to respiratory failure and further increase the risk of pulmonary aspiration [7]. We were concerned that these interactions could potentially be detrimental to our patient's postoperative recovery. We, therefore, decided not to administer any muscle relaxant to facilitate tracheal intubation and using a modified rapid sequence induction with cricoid pressure, performed laryngoscopy and tracheal intubation successfully using sevoflurane and oxygen only.

In conclusion, we describe the first reported anaesthetic for childhood cerebral X-linked adrenoleucodystrophy. In contrast to previous reports, a satisfactory outcome was achieved without the use of muscle relaxant drugs that could potentially have led to postoperative respiratory complications in an already lifelimited patient.

$$
\begin{array}{r}
\text { G. Dobson, J. Lyons } \\
\text { Department of Anaesthesia } \\
\text { The Royal Belfast Hospital for Sick Children } \\
\text { Belfast, N. Ireland }
\end{array}
$$

\section{References}

1. Stephenson DJ, Bezman L, Raymond GV. Acute presentation of childhood adrenoleukodystrophy. Neuropediatrics 2000; 31: 293-297.

2. Kindopp AS, Ashbury T. Anaesthetic management of an adult patient with X-linked adrenoleukodystrophy. Can J Anaesth 1998; 45: 990-992.

3. Schwartz RE, Stayer SA, Pasquariello CA, Lowe DA, Foster CJ. Anaesthesia for the patient with neonatal adrenoleukodystrophy. Can J Anaesth 1994; 41: 56-58.

4. Tobias JD. Anaesthetic considerations for the child with leukodystrophy. Can J Anaesth 1992; 39: 394-397.

5. Cooperman LH. Succinylcholine-induced hyperkalemia in neuromuscular disease. JAMA 1970; 213: 1867-1871.

6. Driessen JJ, Wuis EW, Gielen MJ. Prolonged vecuronium neuromuscular blockade in a patient receiving orally administered dantrolene. Anesthesiology 1985; 62: 523-524.

7. Watson $\mathrm{CB}$, Reierson N, Norfleet EA. Clinically significant muscle weakness induced by oral dantrolene sodium prophylaxis for malignant hyperthermia. Anesthesiology 1986; 65: 312-314.

\section{ERRATUM:}

Reinecke H, Breithardt G, Van Aken H. Cardiological aspects in preoperative anaesthesiological evaluation: old heroes, new shadows. Eur J Anaesthesiol 2003; 20: 595-599.

Table 3, page 596: The first line of the last column reads 'Surgery OK ${ }^{*}$; this is an error and should state 'Further diagnosis'. 